\title{
Thermal and Electrical Properties of Phenol Formaldehyde Foams Reinforcing with Reduced Graphene Oxide
}

Sandhya P K , M S Sreekala ${ }^{\mathrm{b}}$, Abderrahim Boudenne ${ }^{\mathrm{c}}$, Bertrand Garnier ${ }^{\mathrm{d}}$, Didier Rouxel ${ }^{\mathrm{e}}$, Moothetty Padmanabhan ${ }^{\mathrm{a}, \mathrm{f}}$, Nandakumar Kalarikkal' ${ }^{\mathrm{g}}$, Sabu Thomas ${ }^{\mathrm{a}}$

\author{
aSchool of Chemical Sciences, M G University, Kottayam 686560, Kerala, India \\ bPost Graduate Department of Chemistry, Sree Sankara College, Kalady 683574, \\ Kerala, India \\ 'Université Paris-Est, Créteil/ CERTES, 61 Av. du Général de Gaulle, 94010 Créteil \\ cedex, France \\ dLaboratoire de Thermique et Energie de Nantes, UMR CNRS6607, Polytech’ Nantes, \\ Université de Nantes, rue C. Pauc - BP50609 - 44306 Nantes cedex 03, France \\ eInstitut Jean Lamour-UMR CNRS 7198, Facult'e des Sciences et Techniques, Campus \\ Victor Grignard-BP 70239, 54506, Vandoeuvre-les-Nancy Cedex, France \\ fDepartment of Chemistry, Amrita Vishwa Vidyapeetham, Amritapuri, 690525, \\ Kerala, India \\ gInternational and Inter University Centre for Nanoscience and Nanotechonology, M.G. \\ University, Kottayam 686560, Kerala, India
}

\begin{abstract}
In this study, phenol formaldehyde/reduced graphene oxide (PF/RGO) foam nanocomposites were prepared. Here, RGO was obtained by the reduction of graphene oxide using an eco-friendly reducing agent potato starch. The scanning electron microscopic images of RGO reinforced foams exhibited smaller cells with thick cell walls as compared to neat PF foam that confirms the incorporation of filler material. The thermal and dielectric properties of the PF/RGO foams were improved with increasing the wt\% of RGO. The incorporation of RGO improved the thermal conductivity of the PF matrix (11.3\% for $0.15 \mathrm{wt} \%$ of RGO) to a small extent. The prepared foams are efficient thermal insulation materials as well as efficient electrical conductors.
\end{abstract}

Key words: Phenol formaldehyde, reduced graphene oxide, dielectric properties, thermal conductivity 


\section{Graphical Abstract:}

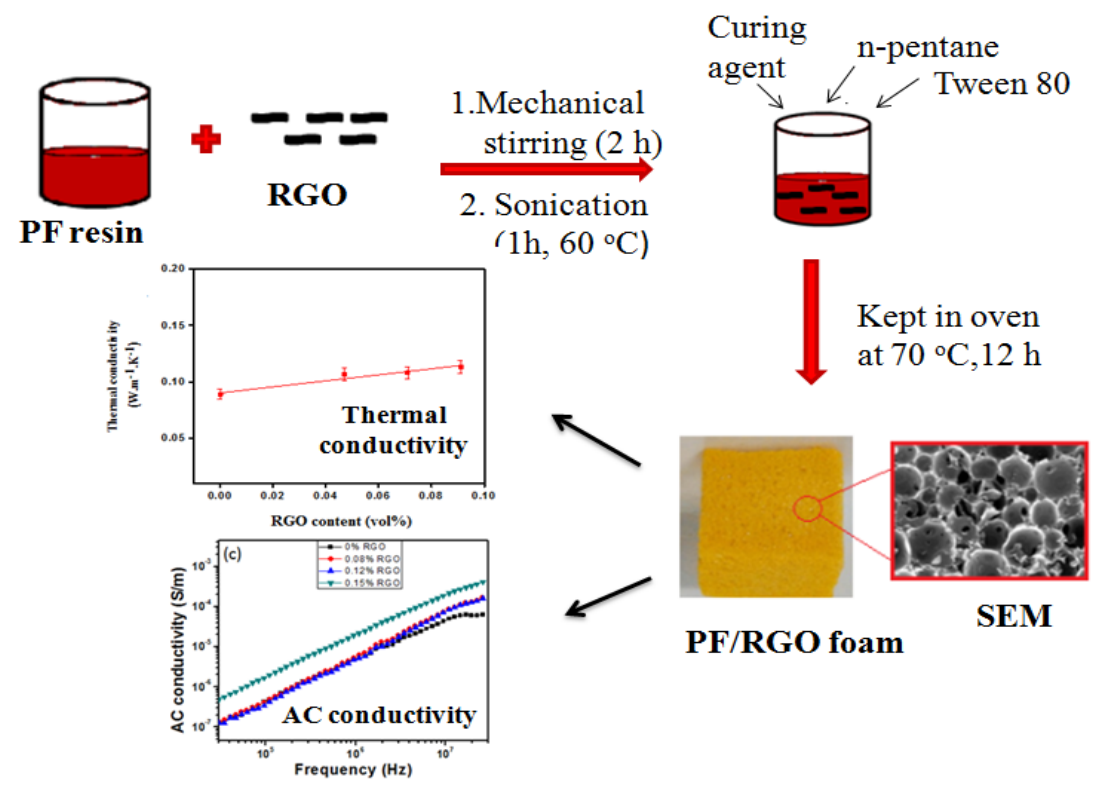

\section{Highlights:}

- RGO obtained by reduction of graphene oxide by potato starch is utilized for the preparation of toughened PF foams

- SEM micrographs illustrated the changes in morphology of PF foam with respect to the nanofiller content

- RGO reinforced phenolic foams showed better thermal stability

- Improvement in electrical conductivity is shown by PF foams with high wt\% RGO

- PF foams remain as thermal insulators as nanocomposites too

\section{Introduction}

Among the polymeric foams, phenolic foam (PF) is readily available and relatively cheap and at the same time it has the greatest FST (Fire, Smoke and less Toxic) properties. Phenolic foams have attained great attention as an insulating material because of its high self-ignition temperature $\left(480{ }^{\circ} \mathrm{C}\right.$ ) and high thermal stability over a broad temperature range (from $-196{ }^{\circ} \mathrm{C}$ to $200{ }^{\circ} \mathrm{C}$ ) [1]. During combustion, it has excellent flame resistance and low generation of toxic gases. Since the phenolic resins contain various solvents, there is no control over the cell morphology during foaming and curing. The cell density and cell uniformity are the factors that affect the physical performance of different polymer foams. The interesting properties of phenolic foams are 
excellent flame retardancy, low smoke density, no dripping of molten plastic during combustion, high resistance to chemicals and solvents etc.

PF foams suffer from lower mechanical properties such as compressive and flexural strengths and high friability and due to these reasons numerous studies have focused on improving these properties [2-4]. Several works have been reported in this field regarding the improvement in the mechanical properties of PF foams [5-9]. One of the main drawbacks of PF foam is its intrinsic brittle nature due to the structure of the molecular chain, which leads to dust pollution in the work place, susceptibility to damage during handling and skin debonding in sandwich structure [10]. The factor that restricts the application of phenolic foam is its brittle nature and powdering [11]. Due to the high friability and low mechanical strength, great efforts have devoted for improving its properties. Improvement in these properties can be achieved by chemical modification, the addition of inert fillers, and fiber reinforcement [12]. Among the different methods, the chemical modification method has gained more attention for its notable toughening effect. Highly flammable foams such as polyurethane and polystyrene can be replaced with phenolic foam for building thermal insulation materials. Toughness is a problem that exists in the field of phenolic foams and it can be improved by adding polyethylene glycol, polyvinyl alcohol, and polyurethane pre-polymer but the flame retardancy is deteriorated because of their flammability $[13,14]$. So, to extend the applications of PF foams, it is important to do some sort of research intensely on toughening agents with improved flame retardancy. Studies on PF foams with aramid fibers [15], glass fibers [16] cardanol [17], boron containing toughening agent [3] were also reported. Traditional PF foams exhibit thermal and electrical insulating property with the closed-cell structure that restricts applications [18]. As a result, it is important to develop PF foams with excellent electrical and electromagnetic interference shielding property by reinforcing conductive nanofillers into the PF matrix.

In the field of material science and condensed-matter physics, graphene is considered as a rising star because of its high electrical conductivity, high surface area, high flexibility, and superior mechanical strength. This two-dimensional (2D) single layer or few layers $\mathrm{sp}^{2}$-bonded graphene has attracted a great deal of interest in recent years owing to its unique structure, cost effective, and extraordinary electronic properties. The enhancement in the texture and homogeneity of foam could be achieved by the addition of inert fillers like talc, mica, clay, glass, and ceramic micro balloons, fly ash etc. This process increases the viscosity of the resin before 
foaming, and thus makes foams heavier. Increase in the foam strength, stiffness, resistance to abrasion and dimensional stability could be obtained by the addition of chopped fibers, including glass fiber, aramid fibers (e.g., Nomex and Kevlar) and natural fibers (bamboo and cellulose fiber). The addition of these fibers affects the mixing and foaming process leading to increased density and poor surface quality. But these results in an enhancement in the mechanical properties of phenolic foam [10].

Seung and colleagues [1] prepared phenolic foams with multi-wall carbon nanotube and graphene and they could improve the mechanical and thermal performance by a short-period microwave foaming process. From the results, they found that $0.5 \%$ MWCNT and $1.0 \%$ graphene (GP) samples show high specific compressive strength with improvement of $71.8 \%$ and $157.3 \%$, respectively. The thermal conductivity of GP 1.0, which has the highest cell density and cell uniformity, decreased by $37.4 \%$ compared with that of the neat phenolic foam and GP 1.0 showed the highest thermal stability from 40 to $390{ }^{\circ} \mathrm{C}$ due to its low thermal conductivity. MWCNTs/phenolic composite foams with three different types of MWCNTs, including pristine, carboxyl and amino MWCNTs were successfully manufactured with the aid of ultrasonic cavitation by Yang et al. [10]. About 31\% increase is observed in the specific strength of foam by the addition of $0.05 \mathrm{wt} \%$ MWCNTs-COOH. It is also observed that a marginal enhancement in thermal stability and flame-retardant property of the composite foam by the addition of various MWCNTs.

$\mathrm{Li}$ and colleagues [19] prepared $\alpha$-zirconium phosphate/graphene oxide and reinforced in phenolic foam and studied mechanical strengths, pulverization ratio, flame retardancy and thermal stability. From the analysis, it was found that there is an increase in flexural and compressive strength and the friability gets decreased. $\mathrm{Li}$ and co-workers [20] synthesized silica nanospheres/graphene oxide hybrid and through immobilization of $\mathrm{SiO}_{2}$ nanospheres on the surface of GO sheets (SGO) and incorporated into PF foams to study its effect on the mechanical strengths, thermal stability, pulverization ratio, and flame retardancy. PF foam with $1.5 \mathrm{phr}$ SGO showed a $36.2 \%$ increase in compressive, a $31.6 \%$ increase in the flexural strength and $70 \%$ decrease in the pulverization ratio. Luan et al. [21] studied the modification of phenolic foam by graphene oxide. From the results, they found that very low percentage of filler can decreased the thermal conductivity of phenolic foam. Zhou and co-workers [22] investigated the effect of graphene oxide on the mechanical properties of phenolic foam, observed an improvement in 
friability and $68.2 \%$ increase of the gel time by using $0.5 \%$ graphene oxide. $\mathrm{Li}$ and co-workers [23] focused on increasing the electrical conductivity as well as the mechanical and thermal insulating properties by introducing the MWCNTs into the PF via in-situ polymerization. From the results, it was found that $\mathrm{PF} / 2 \mathrm{wt} \%$ MWCNTs nanocomposites showed an increase in compressive strength (241.9\%), compressive modulus (166.7\%), tensile strength (264.5\%) and tensile modulus (125.7\%) as compared with that of pure PF.

Saz-Orozco et al. [24] incorporated viscose cellulose fibers into the phenolic foam to improve its compressive mechanical properties. The highest compressive modulus and strength was observed for $2 \mathrm{wt} \%$ cellulose fiber-reinforced phenolic foam and showed an increase of 21\% and $18 \%$, respectively, compared to the unreinforced material. The thermal stability of the phenolic foam gets decreased slightly by the incorporation of cellulose fibers. Liu and co-workers [8] investigated the physical, thermal, flame-retardant and smoke suppression properties of PF foams reinforcing with graphene oxide (obtained by reducing and functionalized with magnesium aminotris-(methylenephophonate). They observed that the pulverization ratio of the samples reduced and enhancement was observed in mechanical strength and flame retardancy. Agrawal et al. [25] developed PF/RGO composite foams by a simple and cost-effective template method. RGO contents in PF foams improved not only the electromagnetic interference shielding effectiveness of composite foams $(-23.2$ to $-50.7 \mathrm{~dB}$ in the X-band frequency region with $1 \mathrm{wt} \%$ RGO) but also thermal (1.2 W/(mK) and electrical properties. Li et al. [26] prepared silica nanoparticle reinforced PF foams using a chemical foaming method to improve their mechanical and thermal properties. The excellent cell morphology and stronger chemical cross-linked network structure of $2 \%$ silica nanoparticle reinforced PF foams showed improvement in compression strength (47.4\%), compression modulus (38.6\%) and tensile strength (42.9\%) compared with the reference PF. TGA results showed that the nanocomposite foams were thermally stable better than pure PF foam. Wang et al. [9] fabricated PF foams with CoAl-layered double hydroxides (LDH)/GO nanohybrid and the resultant samples exhibited an increase in compressive strength (54.8\%), flexural strength (35.2\%) with enhanced thermal stability.

In the present work, in order to avoid the use of a poisonous reducing agent such as hydrazine, an eco-friendly natural reducing agent starch potato was used for the reduction of graphene oxide. The obtained reduced graphene oxide (RGO) was used as a filler in PF for the production of PF/ RGO foam. Here, results from various studies such as morphology, thermal 
stability, dielectric properties, and thermal conductivity of PF/RGO foams are discussed in detail and finally, morphology-property correlations are presented. Hence the applicability of the prepared nanocomposites as a multifunctional material in different fields is exposed.

\section{Experimental}

\subsection{Materials}

Graphite powder, $\mathrm{NaNO}_{3}$, Conc. $\mathrm{H}_{2} \mathrm{SO}_{4}, \mathrm{KMnO}_{4}$, hydrogen peroxide, ammonia, phosphoric acid $\left(\mathrm{H}_{3} \mathrm{PO}_{4}\right.$ ), Tween 80 (polysorbate 80 ), p-toluenesulfonic acid, and n-pentane were all commercial products with analytical grades and were purchased from Merck, India. The starch potato was obtained from LOBA Chemie. Pvt. Ltd. Phenol formaldehyde resole resin was provided by Polyformalin (Ernakulam, Kerala).

\subsection{Preparation of RGO via graphene oxide synthesis}

Reduced graphene oxide was synthesized by reducing graphene oxide (GO) with potato starch. The detailed procedure for the preparation of RGO and its characterization were discussed in our research article published elsewhere [27, 28]. The reduction was carried out by refluxing GO solution using potato starch at $80^{\circ} \mathrm{C}$ for $3 \mathrm{~h}$ by maintaining the $\mathrm{pH}$ in the range of 9-10 through the addition 25\% ammonia solution. The resulting solution washed several times with distilled water and dried at $30^{\circ} \mathrm{C}$ for further use.

\subsection{Preparation of PF/RGO foam nanocomposites}

The weight percentage of RGO for the preparation of PF/RGO foams was taken on the basis of the solid content of the PF resin. For good dispersion of RGO into PF, acetone is used as the solvent and the mixture is stirred in a mechanical stirrer with a speed of 4000 rotations/min for $2 \mathrm{~h}$ and then sonicated for one hour at $60^{\circ} \mathrm{C}$. For $100 \mathrm{~g}$ of PF, n-pentane (blowing agent, $8 \mathrm{~g}$ ), Tween 80 (surfactant, $5 \mathrm{~g}$ ), p-toluenesulfonic acid/phosphorous acid/distilled water in the ratio 2:1:2 by weight (curing agent, $12 \mathrm{~g}$ ) were mixed rapidly at room temperature and is transferred into a paper mould. It was then kept in a pre-heated air oven at $70{ }^{\circ} \mathrm{C}$ for $12 \mathrm{~h}$. The obtained PF and $\mathrm{PF} / \mathrm{RGO}$ foams were cut in desired dimensions for further analysis [19]. A schematic representation of the preparation of PF/RGO foam is shown in Figure 1. 


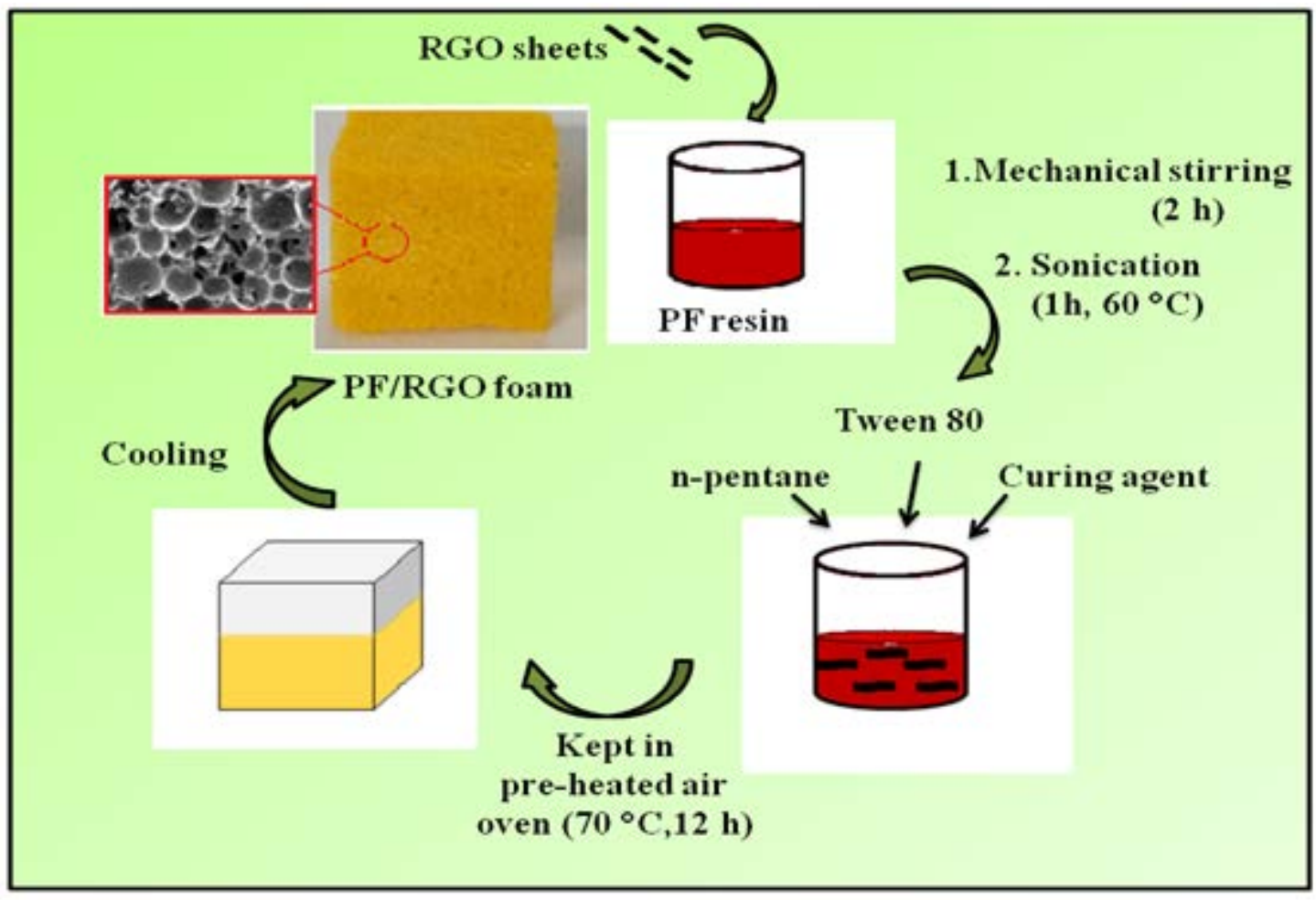

Figure 1. Preparation of PF/RGO foam

\subsection{Characterization of PF/RGO foam nanocomposites}

WITec Alpha 300 RA confocal Raman microscope with AFM (WITec GmbH, Ulm, Germany) is used for measuring the Raman spectra of graphite powder, GO, and RGO. The AFM image of RGO was carried in the same instrument used for measuring Raman spectra. The elemental compositions of the nanomaterials GO, RGO and ZnO-RGO were carried out using energy dispersive X-ray detector OXFORD XMX N model.

The surface morphology of PF/RGO foams was examined using the scanning electron microscope (JEOL JSM-820 model) by directly sticking the solid samples on the conductive tapes.

The thermal stability of the PF foam samples was studied by thermogravimetric analysis (TGA) using Perkin Elmer STA 6000 (Simultaneous Thermal Analyzer) at a heating rate of $20^{\circ} \mathrm{C}$ per minute under nitrogen atmosphere. The weight of the samples used for the analysis was between 5 and $10 \mathrm{mg}$ and the thermograms were recorded from room temperature to $700{ }^{\circ} \mathrm{C}$. 
The dielectric properties of all the samples $\left(10 \times 10 \times 3 \mathrm{~mm}^{3}\right)$ were measured using Wayne Kerr 600B, precision LCR meter with frequency ranging from $20 \mathrm{~Hz}$ to $30 \mathrm{MHz}$. The testing procedure was repeated with four different samples and averaged value was used.

Thermal conductivity $(\lambda)$ was measured at room temperature using a laboratory made experimental device which was designed for the measurement of low and average thermal conductivity from 0.1 to $5 \mathrm{~W} \cdot \mathrm{m}^{-1} \cdot \mathrm{K}^{-1}$ and is suitable for our sample size $\left(10 \mathrm{x} 10 \mathrm{~mm}^{2}\right.$ section and $3 \mathrm{~mm}$ of thickness). The experimental procedure represents an extension of the normalized hot guarded plate (HGP) method with a single-sided specimen state [29, 30]. A heat source and a heat sink (an electrical heater and two heat exchangers with thermostat baths) are located respectively on the bottom and top surfaces of the sample. This setup generates a temperature gradient in the thickness direction of the sample. The main characteristic of the experimental concerns the use of an active guard under the heater that allows the heat flux, dissipated by the electric heater, to flow entirely through the sample. In fact, a temperature controller cancels the heat flux from the electric heater to the heat source. Heat exchanges by adjusting the electric heating in order to obtain its temperature as close as possible to the one of the heat source. The heat exchanger is thus ensuring an adiabatic environment under the heater (Figure 2). The parallelism between upper and lower plates of the experimental setup was achieved using guides for vertical displacement that was controlled by a pneumatic jack in order to ensure an adjustable pressure $P$ on both sides of the sample (typically 4 bar). Thermal resistance between sample and heat source and, between sample and heat sink, were minimized by spreading thermal grease on the top and bottom sides of the sample, the effect of thermal grease on thermal conductivity measurement being previously studied $[29,30]$. When the steady state regime is reached, the measured electric power with respect to the sample surface and the temperatures on both sides of the heater is used to find the thermal conductivity using Fourier's law. This setup which requires only one sample provides thermal conductivity measurement with accuracy smaller than 5\% [30]. 


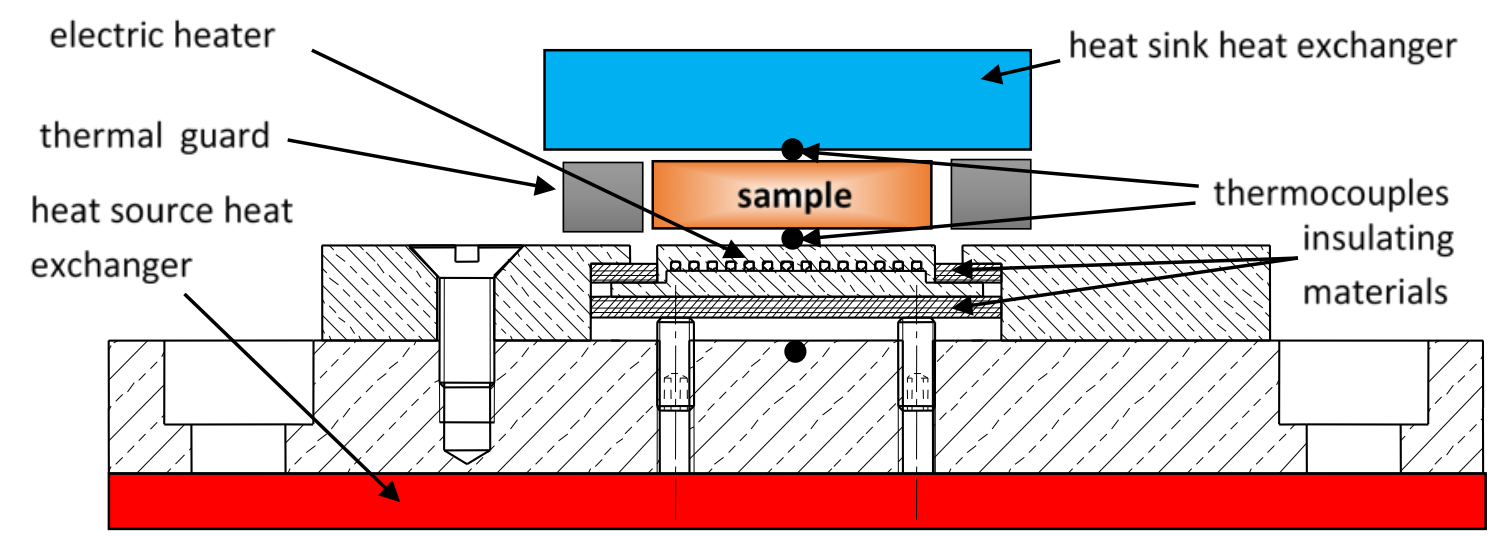

Figure 2. Thermal conductivity experimental setup

Density was found using the sample dimensions and weight, the latter being measured with an A340 Mettler precision balance. The specific heat $(c p)$ measurements were performed using a Perkin Elmer DSC Pyrismicrocalorimeter with a $-70^{\circ} \mathrm{C}$ cryostat. Before heat capacity measurement, the DSC device was calibrated with a $32 \mathrm{mg}$ sapphire reference sample [31].

\section{Results and discussions}

\subsection{Characterization of graphene derivatives}

The Raman spectra patterns of graphite powder, GO, and RGO is represented in Figure 3. Raman spectroscopy analysis was helpful to evaluate the structural properties of graphene based materials. Weak D band observed at $1361 \mathrm{~cm}^{-1}$ in graphite changes to $1369 \mathrm{~cm}^{-1}$ indicating the reduction in the size of the $\mathrm{sp}^{2}$ domains due to the extensive oxidation. After reduction with potato starch, the obtained RGO shows a D band at $1361 \mathrm{~cm}^{-1}$. G band is observed at $1581 \mathrm{~cm}^{-1}$ which corresponds to the vibrations of $\mathrm{sp}^{2}$ carbon atom domains of graphite [32]. If the $\mathrm{G}$ band is present in the Raman spectra, it can be articulated that the sample contains sp ${ }^{2}$ carbon networks [33]. In the case of GO, the broad G band at $1593 \mathrm{~cm}^{-1}$ is due to the presence of isolated double bonds that resonate at much higher frequencies than the $\mathrm{G}$ band of graphite and in the case of RGO it is observed at $1589 \mathrm{~cm}^{-1}$. The ratio of the peak intensities of D and G bands (ID/IG) provides information about the extent of defects on the graphene based materials. The small increase in the D/G intensity ratio of GO (0.9836) and that of RGO (0.9865) indicating that most of the 
oxygenated groups would have been removed during reduction and partially ordered crystal structure of graphene is attained. Similar results were also reported elsewhere [34-36].

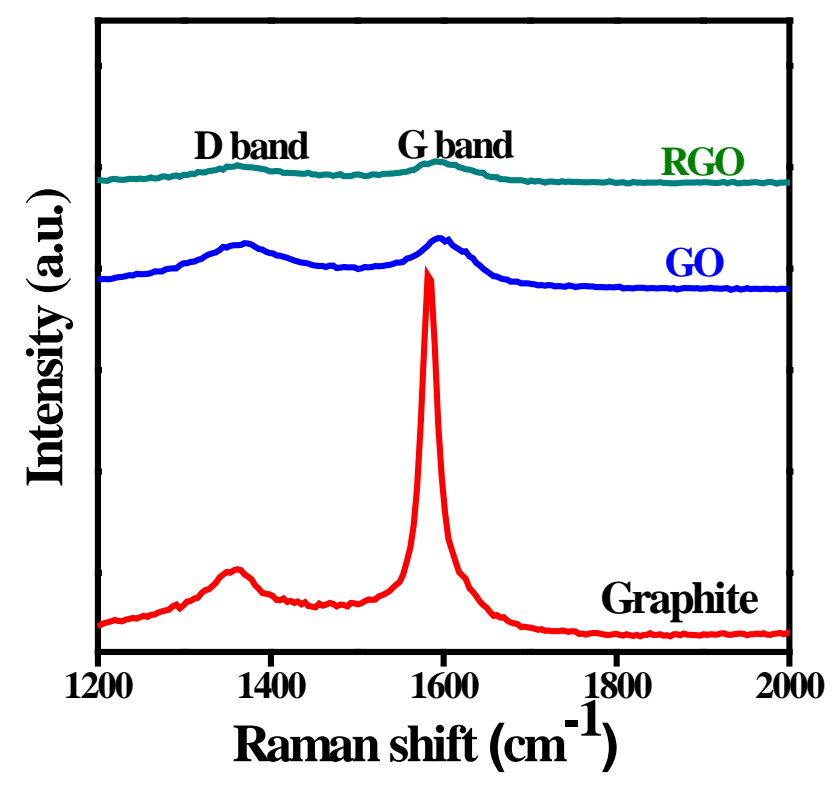

Figure 3. Raman spectra of graphite, GO, and RGO

Figure 4 represents the EDX spectrum of GO and its elemental composition. The spectrum showed peaks corresponding to carbon, oxygen, and sulphur. The presence of trace amount of sulphur in GO is because of the use of $\mathrm{H}_{2} \mathrm{SO}_{4}$ as an oxidizing agent. This result proved the formation of GO. The EDX spectrum of RGO is shown in Figure 4(b) and it is clear that carbon and oxygen is present in it. Moreover, the spectrum corresponding to sulphur is absent in the EDX spectrum of RGO. The increase in the weight percentage of oxygen in RGO (42.06\%) as compared to GO (28.56\%) is due to the presence of oxygen containing functional groups in potato starch. This also confirms that GO is reduced to RGO by potato starch. 

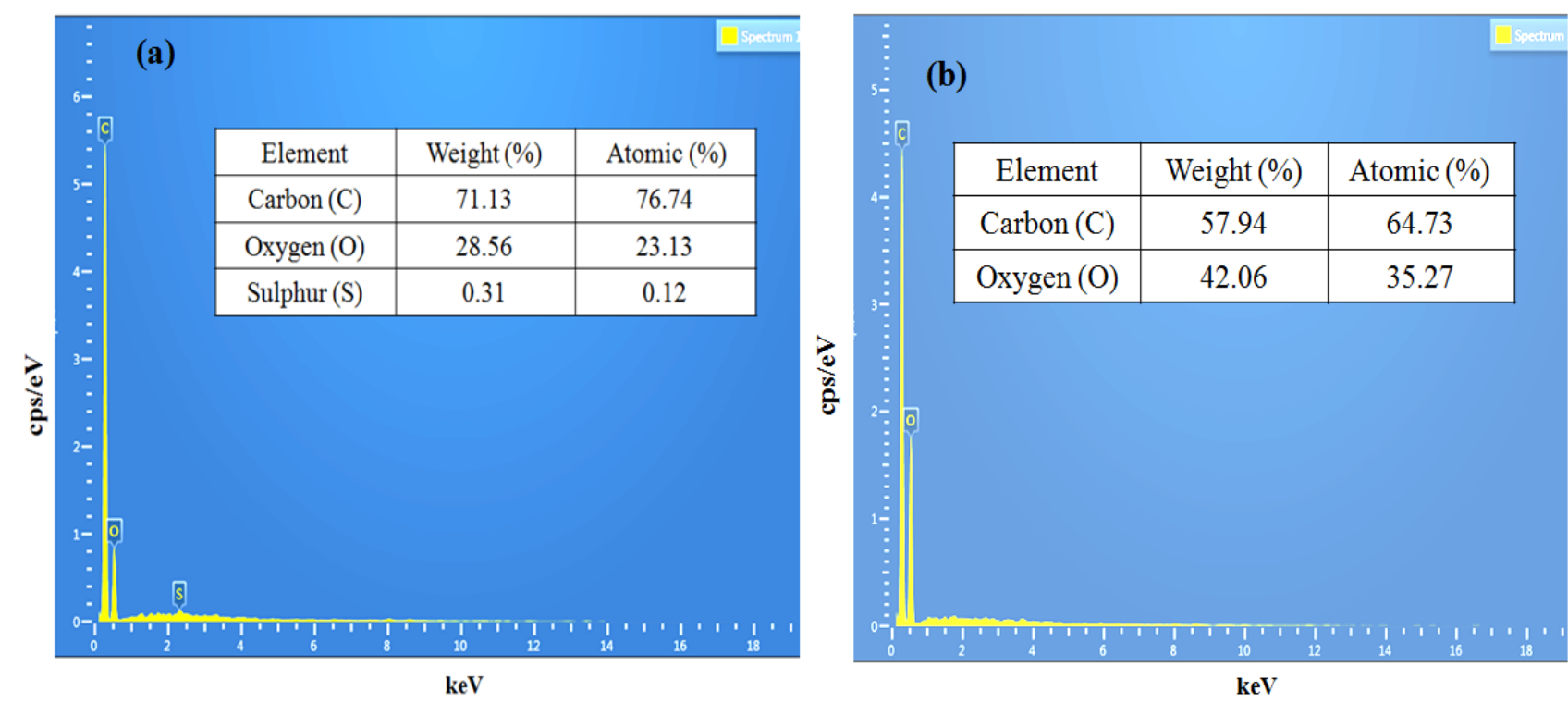

Figure 4. EDX spectra of (a) GO (b) RGO

AFM imaging gives a visualized and more reliable measurement of sheet dimensions such as the thickness of exfoliated graphene sheets. It is difficult to measure the thickness of graphene sheets by AFM measurements due to the surface roughness of graphene sheets and the variations found in the AFM measurement. The normal value of the thickness of single-layer graphene sheets obtained from the AFM images was found to be $1 \mathrm{~nm}$ or less than $1 \mathrm{~nm}$. This may be due to the molecules present on the surface of the graphene sheet, the imperfect interface between the silicon substrate and graphene, and the different attractive forces between AFM probes as compared to the silicon substrate. The thickness of graphene sheets is $2 \mathrm{~nm}$ indicating that it exists as two or three layers. The 3-dimensional AFM image of RGO is obtained by drop casting the dispersion of RGO in water after deposition on a silicon wafer and is represented in Figure 5. From the image, the thickness of RGO sheets is about $0.67 \mathrm{~nm}$ which corresponds to the thickness of single layer graphene sheets $[34,37,38]$. The TEM images of RGO sheets were already published in our previous studies, in which the RGO sheets are irregular and layer structured with wrinkles [28, 39]. 


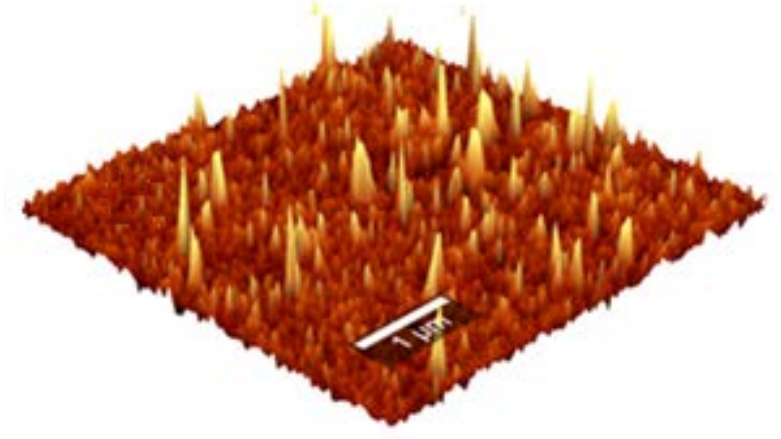

Figure 5. 3-dimensional AFM image of RGO

\subsection{Scanning electron microscopic images of PF/RGO foam nanocomposites}

The cell geometry and the effect of RGO on the microstructure of the PF foams were studied using the SEM analysis. As shown in Figure 6(a), in the case of neat PF, mostly closed cells with large sizes were observed. The walls of some of the cells were broken. With the addition of RGO into the PF matrix, an increase in the number of clear cells was observed in the SEM images as shown in Figure 6(b)-(c). In addition, the shape and size of the cells were almost spherical and the walls of the cells were more rigid than the ones of neat PF. Figure 6(b) shows cells of small and big size distributed randomly with some voids. In Figure 6(c) one can observe clear cells of almost equal shape and size distributed uniformly. This is due to the presence of RGO sheets that embed in the cell walls made them thick, which leads the cells to grow almost similar in size [40]. But on increasing the RGO content, the chances of agglomeration of the RGO sheets increase and the walls of the cells get ruptured as shown in Figure 6(d). Indeed, the SEM image of 0.15 wt\% RGO (Figure 6(d)) shows some collapse or collisions that occurred in the cell. Similar results were reported in studies of Li et al. [40] and Wang et al. [9]. It is observed from the SEM images (Figure 6(a)-(d)) that there is a large numbers of voids present on the surface of samples. This is due to the evaporation of water during the process of foaming and curing. The better interaction of PF with RGO sheets resulted in the formation of almost uniform cells with rigid cell walls. 

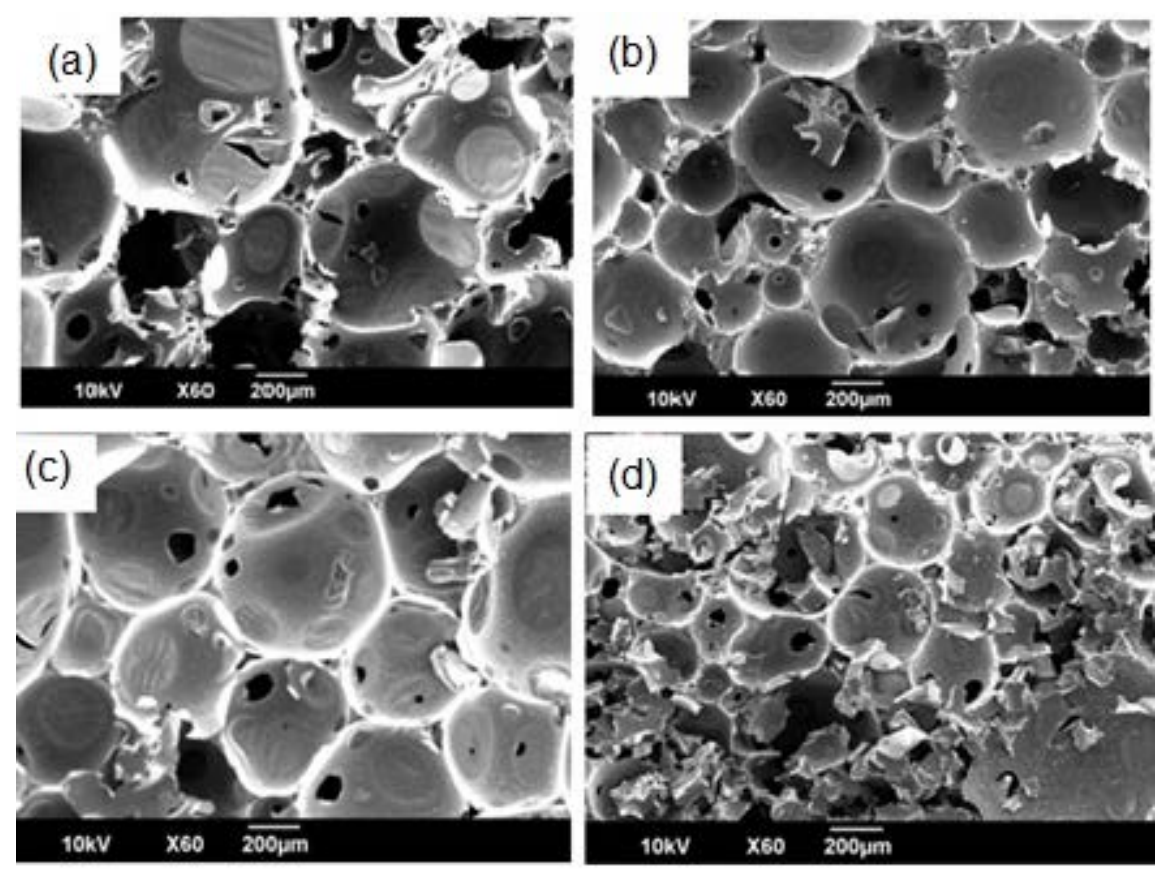

Figure 6. SEM images of (a) neat PF foam (0\% RGO) (b) 0.08 wt\% RGO (c) 0.12 wt $\%$ RGO and (d) $0.15 \mathrm{wt} \% \mathrm{RGO}$

\subsection{Thermal stability of PF foam nanocomposites}

Thermal decomposition of PF and RGO filled PF foams with different weight percentages were studied by TGA and derivative thermogravimetric (DTG) measurements and are shown in Figure 7(a) and (b). Thermograms obtained for PF and PF/RGO foams were almost similar. From the TGA data, it is clear that pure PF foam undergoes decomposition in three stages. The first stage of decomposition occurred in the range $40-100^{\circ} \mathrm{C}$ and is due to the volatilization of blowing agents and moisture present in the foam [19]. The second stage of decomposition occurred in a wide temperature range $100-300{ }^{\circ} \mathrm{C}$, due to the further curing of PF resole, free formaldehyde, phenol and short oligomers and also the decomposition of the surfactant tween $80[9,23]$. The weight loss after $300{ }^{\circ} \mathrm{C}$ is due to the bond cleavage of methylene and aromatic ring. The third stage of decomposition occurred in the range of $400-700{ }^{\circ} \mathrm{C}$ is due to the fracture of the PF chains and further decomposition into low molecular weight products. A fast fall in TG curve is observed when the temperature continuous to creep from 430 to $580{ }^{\circ} \mathrm{C}$. The addition of RGO restricts the molecular chain mobility of the PF to some extent and leads to a decrease in the rate of decomposition. Generally, the modified foams have better thermal stability than neat PF throughout the entire temperature range. 

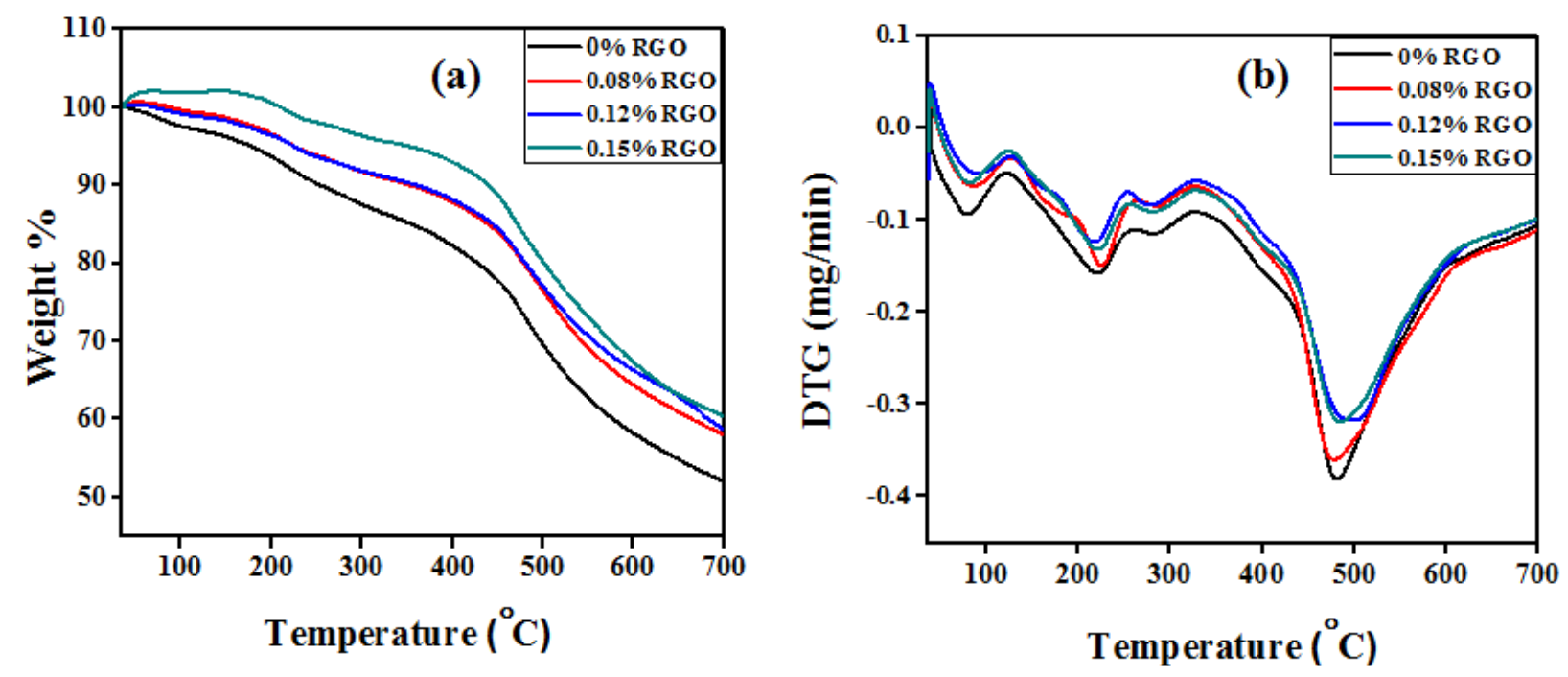

Figure 7. (a) TGA and (b) DTG of PF/RGO foams

From the DTG results, it is clear that there are three DTG peaks located approximately at $80{ }^{\circ} \mathrm{C}, 221{ }^{\circ} \mathrm{C}$ and $482{ }^{\circ} \mathrm{C}$ corresponding to the evaporation of water, dehydration due to further curing and the degradation of phenolic resin, respectively. The degradation temperatures at $5 \%$ and $10 \%$ loss and the temperature of the maximum decomposition rate $\left(\mathrm{T}_{\max }\right)$ in DTG peaks, and char residue at $700^{\circ} \mathrm{C}$ are listed in Table 1 . As compared to neat PF, a slight increase in degradation temperature is observed for PF/RGO foams. The char residue at $700{ }^{\circ} \mathrm{C}$ of $\mathrm{PF} / \mathrm{RGO}$ foams gradually increased with increasing the RGO content as evident from Table 1 . The char residue obtained at $700{ }^{\circ} \mathrm{C}$ for $0.15 \%$ RGO shows $16.14 \%$ increase as compared to neat PF. PF/RGO foam with $0.15 \%$ shows the highest value for $\mathrm{T}_{-5 \%}, \mathrm{~T}_{-10 \%}$ and highest char residue at $700{ }^{\circ} \mathrm{C}$ indicating the highest thermal stability. The network of RGO sheets in the PF matrix affects the heat and mass transfer in phenolic foams. From these results, it is clear that there is an improvement in the thermal stability of the PF foams with the incorporation of RGO into the PF matrix.

Table 1. Degradation temperatures of PF and PF/RGO foams

\begin{tabular}{|c|c|c|c|c|}
\hline Sample code & $\mathrm{T}_{-5 \%}\left({ }^{\circ} \mathrm{C}\right)$ & $\mathrm{T}-10 \%\left({ }^{\circ} \mathrm{C}\right)$ & $\mathrm{T} \max \left({ }^{\circ} \mathrm{C}\right)$ & $\begin{array}{c}\text { Char residue } \\
(\%) \\
\text { at } 700^{\circ} \mathrm{C}\end{array}$ \\
\hline $0 \%$ RGO & 177.83 & 254.86 & 482.82 & 51.99 \\
\hline $0.08 \%$ RGO & 225.54 & 352.97 & 480.70 & 57.99 \\
\hline $0.12 \%$ RGO & 225.41 & 359.94 & 502.17 & 58.73 \\
\hline $0.15 \%$ RGO & 350.45 & 440.75 & 483.22 & 60.38 \\
\hline
\end{tabular}

\subsection{Electrical properties of phenolic foam nanocomposites}


The electrical property of phenol formaldehyde foam with different wt\% of RGO is shown in Figure 8 and it is observed that the dielectric permittivity, dielectric loss and AC conductivity of PF/RGO foams gradually increased with increase in the wt\% of RGO. Here, only a small increase in conductivity is observed at lower wt\% RGO.
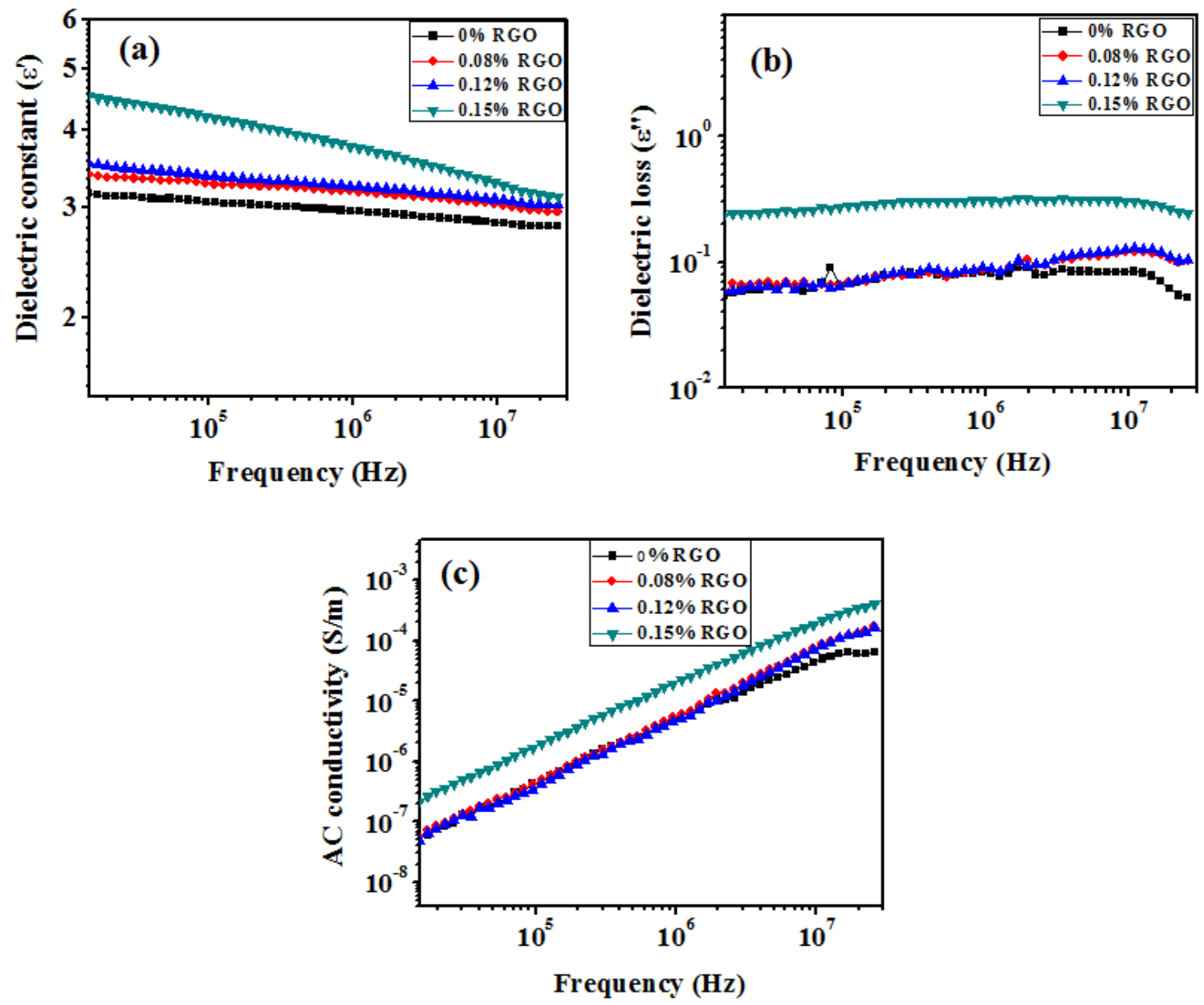

Figure 8. Dielectric properties of PF/RGO foams

From Figure 8(a), it can be seen that the dielectric constant of PF/RGO foams increased dramatically at $0.15 \mathrm{wt} \%$ RGO because, at higher concentration of RGO the possibility for the formation of conductive networks increases as a result of the accumulation of a large number of graphene sheets in the cell walls of the foam. Here, foams exhibit a significant percolation property when the RGO content reach $0.15 \mathrm{wt} \%$, due to the formation of the conductive path of the interconnected RGO sheets. Narasimman et al. studied the electrical conductivity in GO based carbon foams and found that higher wt\% of GO (1.25\%) showed an increase in electrical 
conductivity (4.8 S cm $\mathrm{S}^{-1}$ ) [41]. Figure 9 represents the conductivity network formed by RGO sheets in PF foam which cause electrical conductivity.

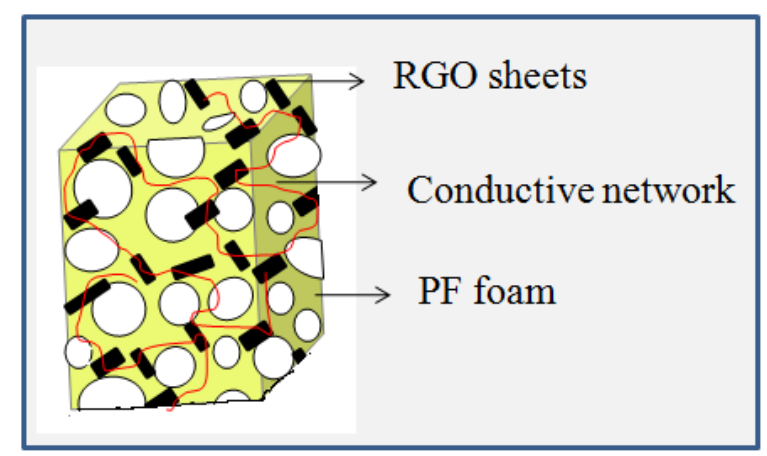

Figure 9. PF foam with RGO sheets

\subsection{Thermophysical properties of PF/RGO foam nanocomposites}

The investigation of thermal conductivity of PF/RGO foams is essential when it plays a very important role in its application. In the case of foams, the heat transfer mainly occurred through the solid polymer than through the gaseous phase. The density, size, and distribution of the cells, the thermal conductivity of the polymer etc. are some of the factors that affect the thermal conductivity of foams [29]. The relationship between the thermal conductivity of the resulting foams and RGO volume content is shown in Figure 10.

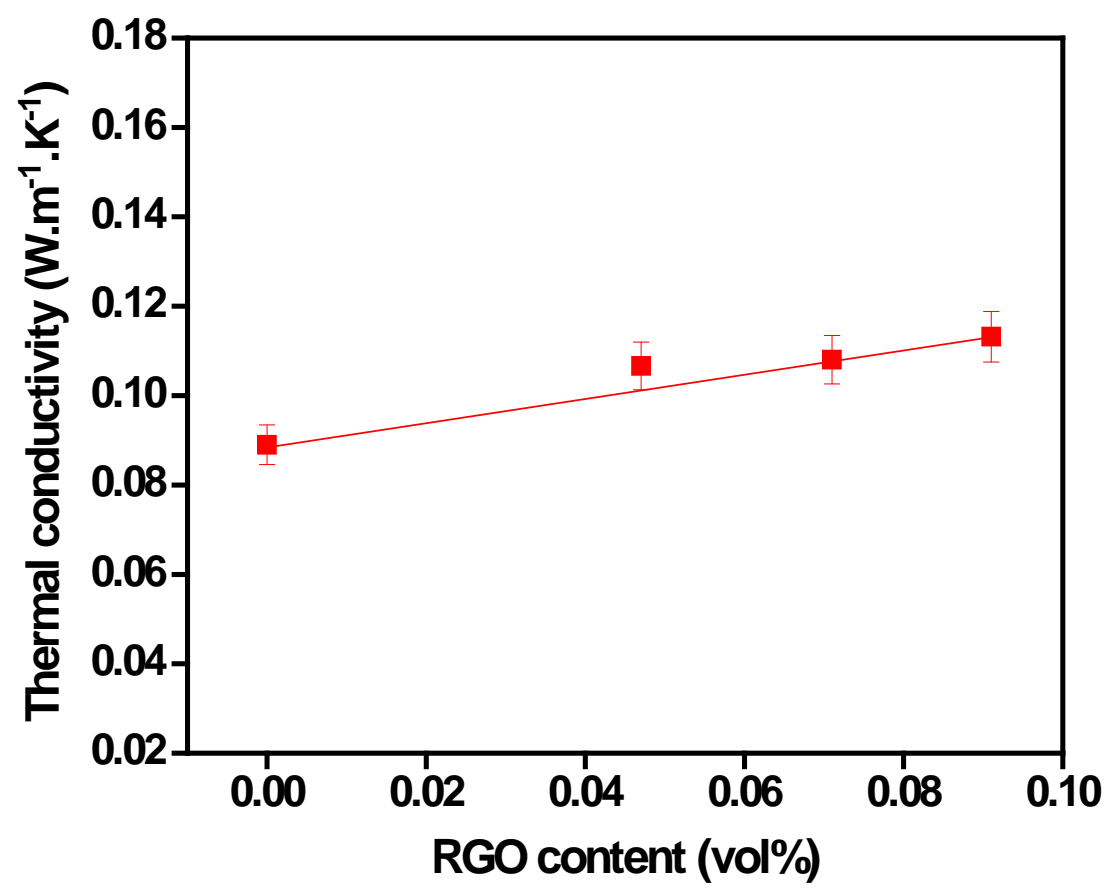


Figure 10. Thermal conductivity of PF/RGO foams

The evolution of the thermal conductivity of composite materials is almost presented in vol\% because of the predicted and theoretical models are expressed in volume content. From figure, it is clear that the thermal conductivity of PF/RGO foams shows a slight increase in thermal conductivity with an increase in vol\% of RGO. The thermal conductivity of neat PF foam is 0.089 $\mathrm{W} \cdot \mathrm{m}^{-1} \cdot \mathrm{K}^{-1}$ and the maximum value $\left(0.113 \mathrm{~W} \cdot \mathrm{m}^{-1} \cdot \mathrm{K}^{-1}\right)$ is observed for PF/RGO foam with $0.9 \mathrm{vol} \%$ RGO which corresponds to $0.15 \mathrm{wt} \%$ RGO. The slight increase in thermal conductivity of PF/RGO foams is due to the presence of RGO which creates thermal paths through the solid PF. From the results, it can be conclude that the addition of RGO in phenolic foams did not produce any dramatic change in the insulating property of PF foam.

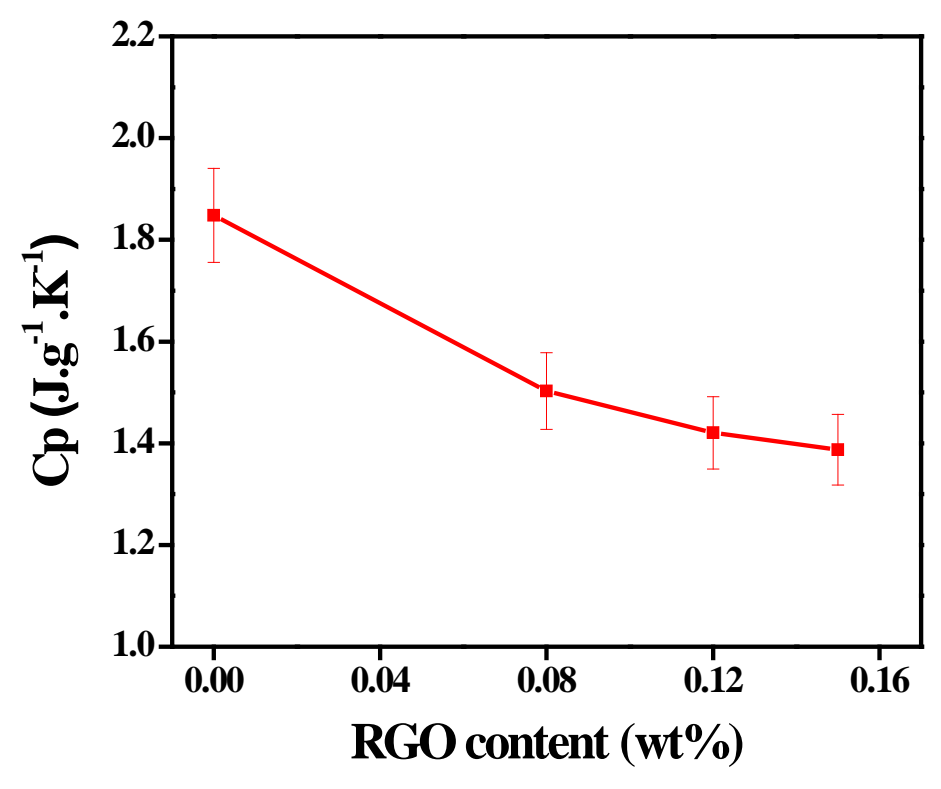

Figure 11. Specific heat of PF/RGO foams

Figure 11 shows the specific heat of PF/RGO foams. According to the results, the specific heat of PF/RGO foams decreases with an increase in RGO content. The specific heat of neat PF is $1.848 \mathrm{~J} \cdot \mathrm{g}^{-1} \cdot \mathrm{K}^{-1}$, and decreases with the adding of RGO due to the smaller heat capacity of RGO (around 720 J.kg-1.K-1) therefore the minimum value of specific heat is observed for $0.15 \mathrm{wt} \%$ RGO. Here, the reinforcement of RGO causes a small change in the heat capacity of PF/RGO foams. The thermophysical measurements of PF/RGO foams with varying wt\% RGO are summarized in Table 2 . 
Table 2. Thermophysical properties of PF/RGO foams (at $25^{\circ} \mathrm{C}$ )

\begin{tabular}{|c|c|c|c|}
\hline Sample & $\begin{array}{c}\text { Thermal conductivity } \\
\lambda \\
\left(\mathrm{W} \cdot \mathrm{m}^{-1} \cdot \mathrm{K}^{-1}\right)\end{array}$ & $\begin{array}{l}\text { Heat capacity } \\
\mathrm{Cp}\left(\mathrm{J} \mathrm{g}^{-1} \cdot \mathrm{K}^{-1}\right)\end{array}$ & $\begin{array}{l}\text { Density } \\
\left(\mathrm{kg} \cdot \mathrm{m}^{-3}\right)\end{array}$ \\
\hline 0\% RGO & 0.089 & 1.848 & 58.80 \\
\hline $0.08 \%$ RGO & 0.107 & 1.503 & 84.90 \\
\hline $0.12 \%$ RGO & 0.108 & 1.420 & 60.00 \\
\hline $0.15 \%$ RGO & 0.113 & 1.387 & 70.00 \\
\hline
\end{tabular}

Each density value from Table 2 is the average of four measurements on four different samples showing relative standard deviations less than $9 \%$ which are smaller than the discrepancies shown in the Table 2 between densities with various RGO contents. Therefore the observed effects of RGO content on density are due to some phenomenon. Indeed, the addition of $0.08 w t \%$ RGO decreases the size of the cell foam as observed in section 3.2 dedicated to SEM observations. Therefore, the density increases with RGO content and also because of microstructure change of the foam. As further amount of RGO is added, some destruction of cell walls is observed (section 3.2) which could explain the decrease of density with the 0.12 and $0.15 w t \%$ of RGO. The heat capacity of PF/RGO is affected by the addition of RGO but not by the microstructure change of the foam as it is a thermal property related to the mass. The change of microstructure of the foam could affect the thermal conductivity however this effect is hidden by the increase in effective thermal conductivity due to the addition of RGO material which has a much higher thermal conductivity (typically about $6 \mathrm{~W} \cdot \mathrm{m}^{-1} \cdot \mathrm{K}^{-1}$ ) than PF material.

\section{Conclusions}

In our work, PF foams were reinforced using RGO and resulting morphology, thermal stability, dielectric property and thermal conductivity of the foams were studied. The SEM images of PF/RGO foams show changes in the size and distribution of the cells with an increase in RGO content. PF foams with 0.12 wt\% RGO shows cells of uniform size and shape. From the TGA results, it is found that there is an improvement in the thermal stability of PF foams. The dielectric properties of the PF foams increase with an increase in wt\% of RGO. The thermal conductivity study of the prepared foams shows that the introduction of RGO into the PF matrix increases 
slightly the thermal conductivity of the foams. Here, the samples remained as thermal insulators. On analyzing the thermal conductivity and dielectric properties of PF foams, one could expect applications of this material when relatively electrical conductor and thermal insulating properties are both required.

\section{Acknowledgements}

The authors are grateful to the financial support under DST-FIST (No. 487/DST/FIST/15-16) New Delhi to Sree Sankara College, Kalady and Mahatma Gandhi University, Kottayam, India for the award of Junior Research Fellowship for Sandhya P K.

\section{References}

1. S. A. Song, Y. S. Chung, S. S. Kim, Compos. Sci. Technol. 2014, 103, 85.

2. M. Gao, Y.L. Yang, Z.Q. Xu, Adv. Mater. Res.2013, 803, 21.

3. L. Liu, M. Fu, Z. Wang, Ind. Eng. Chem. Res. 2015, 54, 1962.

4. X. Sui, Z. Wang, Polym. Advan. Technol. 2013, 24, 593.

5. M. Gao, W. Wu, Y. Wang, Y. Wang, H. Wang, J. Therm. Anal. Calorim. 2016, 124, 189.

6. Y. Ma, X. Geng, X. Zhang, C. Wang, F. Chu, Pol. J. Chem. Technol. 2018, 20, 47.

7. L. Hu, Z. Wang, Q. Zhao, Polym. Plast. Technol. Eng. 2017, 56, 678.

8. L. Liu, Z. Wang, J. Hazard. Mater. 2018, 357, 89.

9. Z. Wang, X. Li, High Perform. Polym. 2018, 30, 688.

10. Z. Yang, L. Yuan, Y. Gu, M. Li, Z. Sun, Z. Zhang, J. Appl. Polym. Sci. 2013, 130, 1479.

11. H. Yang, X. Wang, B. Yu, H. Yuan, L. Song, Y. Hu, R. K. Yuen, G. H. Yeoh, J. Appl. Polym. Sci. 2013, 128, 2720.

12. Q. Xu, R. Gong, M. Cui, C. Liu, R.H. Li, High Perform. Polym. 2015, 27, 852.

13. H. Yang, X. Wang, H. Yuan, L. Song, Y. Hu, R.K. Yuen, J. Polym. Res. 2012, 19, 9831.

14. T. Horikawa, K. Ogawa, K. Mizuno, J.I. Hayashi, K. Muroyama, Carbon, 2003, 41, 465.

15. H. Shen, S. Nutt, Compos. Pt. A-Appl. Sci. Manuf, 2003, 34, 899.

16. H. Yuan, W. Xing, H. Yang, L. Song, Y. Hu, G.H. Yeoh, Polym. Int. 2013, 62, 273.

17. S. Jing, T. Li, X. Li, Q. Xu, J. Hu, R. Li, J. Appl. Polym. Sci. 2014, 131.

18. Q. Li, L. Chen, J. Ding, J. Zhang, X. Li, K. Zheng, X. Zhang, X. Tian, Carbon. 2016, 104, 90. 
19. X. Li, Z. Wang, L. Wu, T. Tsai, RSC Adv. 2016, 6, 74903.

20. X. Li, Z. Wang, L. Wu, RSC Adv. 2015, 5, 99907.

21. J. P. Luan, X. F. Wei, X. P. Zhou, G. Z. Lu, C. Z. Liu, G. D. Li, Y. X. Na, H. W. Wang, X. W. Qi, J. Adv. Mater. Res. 2015, 1095, 510.

22. J. Zhou, Z. Yao, Y. Chen, D. Wei, T. Xu, Polym. Compos. 2014, 35, 581.

23. Q. Li, L. Chen, X. Li, J. Zhang, X. Zhang, K. Zheng, F. Fang, H. Zhou, X. Tian, Compos. Part A. Appl. Sci. Manuf. 2016, 82, 214.

24. B. Del Saz-Orozco, M.V. Alonso, M. Oliet, J.C. Domínguez, F. Rodriguez, Compos. Part B. Eng. 2015, 75, 367.

25. P.R. Agrawal, R. Kumar, S. Teotia, S. Kumari, D.P. Mondal, S.R. Dhakate, Compos.Part B. Eng. 2019, 160, 131.

26. Q. Li, L. Chen, J. Zhang, K. Zheng, X. Zhang, F. Fang, X. Tian, Polym. Eng. Sci. 2015, 55, 2783.

27. W. S.Hummers Jr, R. E. Offeman, J. Am. Chem. Soc. 1958, 80, 1339.

28. P. K. Sandhya, J. Jose, M. S. Sreekala, M. Padmanabhan, N. Kalarikkal, S. Thomas, Ceram. Int. 2018, 44, 15092.

29. R. Tlili, A Boudenne, V. Cecen, L. Ibos, I. Krupa, Y. Candau, Int. J. Thermophys. 2010, 31, 936.

30. F. Danes, B. Garnier, T. Dupuis, Int. J. Thermophys. 2003, 24, 771.

31. A. Boudenne, L. Ibos, M. Fois, J. C. Majesté, E. Géhin, Compos. Part A. Appl. Sci. Manuf. 2005, 36, 1545.

32. X. Zhao, Y. Li, J. Wang, Z. Ouyang, J. Li, G. Wei, Z. Su, ACS Appl. Mater. Interfaces, 2014, 6, 4254.

33. M.A. Pimenta, G. Dresselhaus, M.S. Dresselhaus, L.G. Cancado, A. Jorio, R. Saito, Phys. Chem. Chem. Phys. 2007, 9, 1276.

34. Y. Feng, N. Feng, G. Du, RSC Adv. 2013, 3, 21466.

35. S. Sadhukhan, T.K. Ghosh, D. Rana, I. Roy, A. Bhattacharyya, G. Sarkar, M. Chakraborty, D. Chattopadhyay, Mater. Res. Bull. 2016, 79, 41.

36. Z. Bo, X. Shuai, S. Mao, H. Yang, J. Qian, J. Chen, J. Yan, K. Cen, Sci. Rep. 2014, 4, 4684. 37. X. Wang, W. Xing, B. Yu, X. Feng, L. Song, Y. Hu, J. Mater. Chem. C. 2013, 1, 690. 
38. Q. Zhuo, J. Gao, M. Peng, L. Bai, J. Deng, Y. Xia, Y. Ma, J. Zhong, X. Sun, Carbon. 2013, 52, 559.

39. P.K. Sandhya, M.S. Sreekala, M. Padmanabhan, K. Jesitha, S. Thomas, Compos. B. Eng. 2019, 167, 83.

40. Q. Li, L. Chen, X. Li, J. Zhang, K. Zheng, X. Zhang, X. Tian, J. Appl. Polym. Sci. 2016, 133, 43765.

41. R. Narasimman, S. Vijayan, K. Prabhakaran, J. Mater. Sci. 2015, 50, 8018. 